\title{
Компьютерное моделирование межчастичных взаимодействий в фазе анионообменника при необменной сорбции аминокислот
}

\author{
Трунаева Е.С., Хохлова О.Н., Хохлов В.Ю. \\ ФГБОУ ВО «Воронежский государственный университет». Воронеж
}

Поступила в редакцию 8.02.2018 г.

DOI: https://doi.org/10.17308/sorpchrom.2018.18/493

Исследованы межчастичные взаимодействия в фазе анионообменника AB-17в Cl-форме при необменном поглощении биполярных ионов глицина, фенилаланина и триптофана. Квантовохимическое моделирование образующихся структур проводили с помощью программы Gaussian 03 методом гибридного функционала плотности B3LYP в базисе 6-31G++(d,p). Показано, что усложнение строения радикала аминокислоты приводит к уменьшению выигрыша энергии сорбции.

Ключевые слова: квантово-химическое моделирование, глицин, фенилаланин, триптофан, высокоосновный анионообменник, необменное поглощение.

\section{Computer simulation of interparticle interactions in the anion exchanger phase at non-exchange sorption of amino acids}

\author{
Trunaeva E.S., Khokhlova O.N., KhokhlovV.Yu. \\ Voronezh State University, Voronezh
}

\begin{abstract}
At theabsence in the sorption system of the main operating force, for example, of Coulomb interaction between the functional group and the counter ion in the ion exchanger, sorption of substances proceeds through of the specific forces of various nature comparable in size. The existence of features in a structure of sorbate can lead to the appearance of various effects strengthening or weakening interactions in system. Such effects should be expected in sorption systems involving amino acids. Existence in each bipolar of aminoacid group defines identity of their fixing in a sorbent and the difference in a structure of the radical will determine features of the sorption of each amino acid.

Intermolecular interactions between glycine, phenylalanine and tryptophane from water solutions anda AV-17-2P anion exchanger ( $\mathrm{Cl}$ - form) is investigated. The presence in solution of bipolar ions of amino acids and impossibility of their recharge in the sorbent phase to anion with the subsequent fixing about functional groups as a result of Coulomb interaction leads to implementation of non exchange sorption of amino acids. The purpose of work is establishing of interactions acting in system at non exchange sorption of amino acids by the anion exchanger and to reveal of lateral radical's natureat forming ion-molecular structures in an ion exchanger phase.

Computer modeling of structures and calculation of energies using the Gaussian 03 program implementing the B3LYP hybrid densityfunctional with the $6-31 \mathrm{G}++(d, p)$ basis set. Starting fragments of the hydrated amino acids and the anion exchanger placed variously from each other and optimized considered structure with the smallest energyare made.

The main acting forces in the sorbent phase at non exchange sorption of amino acids are an ion - ion and an ion - dipole interactions of the functional group of the sorbent, chlorine counterion, a bipolar ion with each other and water molecules. At complication of the structure of the amino acid'slateral radical in the
\end{abstract}


sorption systems with the mineral counterion of AV-17 (Cl) $+\mathrm{AK} \pm$, structural characteristics and energy gain are slightly changes.

Keywords: quantum chemical simulation, glycine, phenylalanine, tryptophane, high basic anion exchanger, non-exchange sorption.

\section{Введение}

При отсутствии в сорбционной системе основной действующей силы, например, кулоновского взаимодействия между функциональной группой и противоионом при ионом обмене, поглощение веществ протекает за счет специфических сил различной природы, сопоставимых по энергии. При этом наличие особенностей в строении сорбата может приводить к возникновению различных эффектов, усиливающих или ослабляющих взаимодействия в системе. Такие эффекты следует ожидать в сорбционных системах с участием аминокислот. Наличие в каждом сорбате аминокислотной группировки определяет идентичность их закрепления в сорбенте, а различие в строении радикала будет определять особенности закрепления каждой аминокислоты [1-3].

В работе исследованы межмолекулярные взаимодействия в фазе сорбента при необменной сорбции глицина, фенилаланина и триптофана из водных растворов анионообменником AB-17-2П в Cl-форме. Наличие в растворе биполярных ионов аминокислот и невозможность их перезарядки в фазе сорбента в анион с последующим изменением ионной формы сорбента, приводит к протеканию необменного поглощения цвиттерлитов [3-5]. Целью работы являлось установление природы сил, действующих в системе при необменной сорбции аминокислот анионообменником и выявление роли природы бокового радикала при формировании ионномолекулярных структур в фазе ионообменника.

\section{Эксперимент}

Компьютерное моделирование структур и вычисление энергий проводились с использованием программы Gaussian 03 методом гибридного функционала плотности B3LYP в базисе $6-31 \mathrm{G}++(\mathrm{d}, \mathrm{p})$. Этот базис включает в расчет $p$-орбитали атомов водорода и $d$-орбитали $p$-элементов (валентно-расщепленный базис), что существенно повышает точность расчета [6]. Стартовые фрагменты гидратированных аминокислот и анионообменника располагали различным образом друг относительно друга и оптимизированной считали структуру с наименьшей энергией.

\section{Обсуждение результатов}

Высокоосновные анионообменники типа АВ-17 являются стиролдивинилбензольными сорбентами, имеющими в качестве функциональных групп четвертичный азот (рис.1).

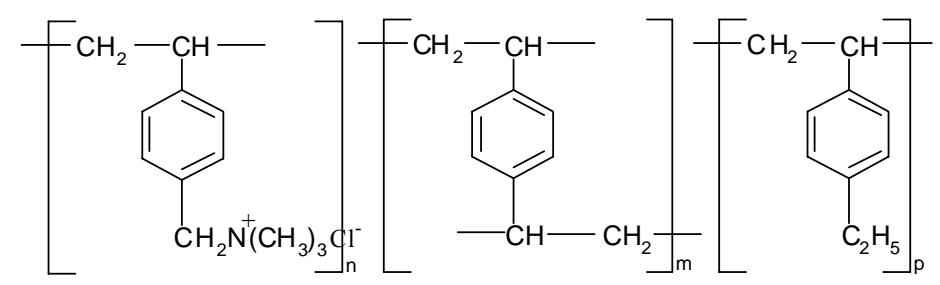

Рис. 1. Строение составного повторяющегося звена анионообменника AB-17 [7] 
Анионообменники широко используются для очистки воды различного назначения, разделения и очистки физиологически активных веществ [8,9]. Моделирование анионообменников освещено в литературе существенно меньше, чем катионообменников, однако имеются работы по квантово-химическим расчетам их структуры при наличии в системе различных неорганических противоионов, некоторых поглощенных органических веществ, а также в составе мембран [10-13], где несмотря на различные использованные методы и базисы моделирования, представлена идентичная структура и гидратация составного повторяющегося звена. Однако для корректного дальнейшего рассмотрения взаимодействия анионообменника с аминокислотами нами проведено моделирование структуры элементарного звена анионообменника в Cl-форме с последовательной гидратацией одной - восемью молекулами воды. Стартовым фрагментом анионита выбран элемента матрицы, содержащий бензольное кольцо и четвертичный азот в качестве функциональной группы. На рис. 2 представлена оптимизированная структура, содержащая пять молекул $\mathrm{H}_{2} \mathrm{O}$.

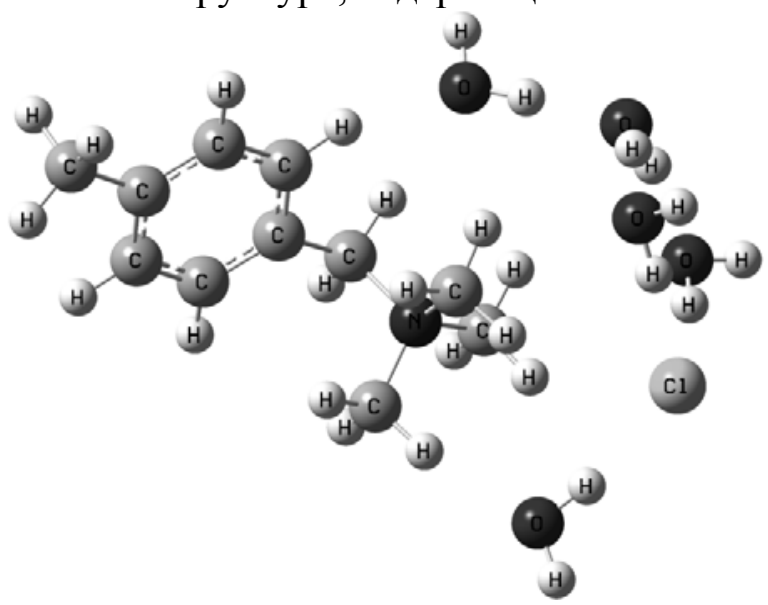

Рис. 2. Оптимизированная гидратированная структура фрагмента AB-17 ( $\mathrm{HCl})$ с пятью молекулами воды

Как видно, молекулы воды формируют первичную гидратную оболочку иона хлора и группы $-\mathrm{N}^{+}\left(\mathrm{CH}_{3}\right)_{3}$, расстояние между противоионом и атомом азота функциональной группы составляет $4.42 \AA$, при этом элемент углеводородной матрицы ионообменника остается негидратированным.

Квантово-химическое моделирование гидратации и ассоциации аминокислот в водных растворах, а так же на границах раздела фаз и при образовании пептидов и белков исследовано ранее [14-18]. Показано, что гидратная оболочка карбоксильной группы аминокислот содержит четыре молекулы воды, а аминогруппа - три молекулы $[14,17,18]$. Нами проведена оптимизация ионно-молекулярных структур, образующихся в сорбенте при необменной сорбции биполярных ионов аминокислот с гидрофобным радикалом - фенилаланина и триптофана. В качестве «аминокислоты сравнения» выбран глицин -простейшая аминокислота, не имеющая бокового радикала. Для квантово-химических расчетов использовали стартовые фрагменты анионообменника и аминокислоты, содержащие по пятьмолекул воды. Суммарное количество растворителя $\left(10 \mathrm{H}_{2} \mathrm{O}\right)$ обеспечит формирование гидратных оболочек каждого компонента системы, которые, однако, будут иметь общие молекулы воды, т.е. перекрываться.

На рис. 3 представлены оптимизированные структуры, образующиеся в фазе сорбента. Как видно из рисунка, аминокислоты ориентируются положительно заряженной аминогруппой к противоиону хлора, как и описано в работах по исследованию равновесия необменной сорбции $[4,5]$. Гидратная вода образует единую систе- 
му, располагаясь между положительными и отрицательными структурными элементами системы, при этом у фенилаланина и триптофана гидрофобные радикалы остаются негидратированными, как и элемент матрицы сорбента. Поскольку фаза сорбента является очень концентрированной, ароматические структуры матрицы и аминокислоты находятся на очень малом расстоянии друг от друга, достаточном для реализации $\pi$ - $\pi$ взаимодействий [19], которые описываются в литературе $[20,21]$, однако, расположение стартовых фрагментов ароматическими структурами друг к другу для реализации гидрофобных взаимодействий, не является энергетически выгодным и не приводит к получению оптимизированных структур. Поэтому, можно говорить, что доминирующими силами при закреплении необменно поглощенной аминокислоты в сорбенте являются полярные ион-ионные и ион-дипольные взаимодействия заряженной функциональной группы сорбента, противоиона хлора, биполярного иона цвиттерлита друг с другом и молекулами воды.

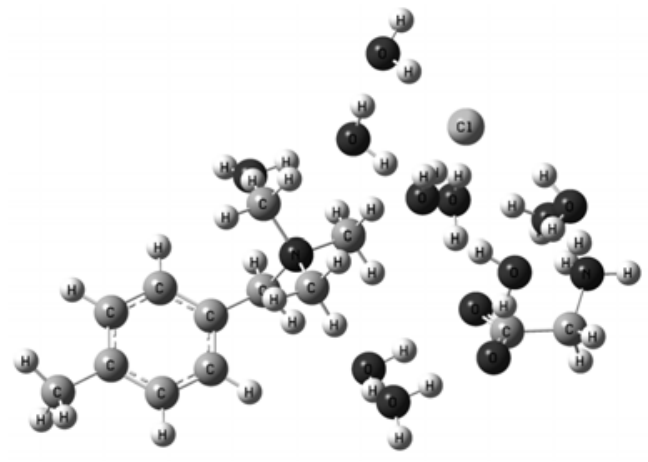

$\mathrm{a}$

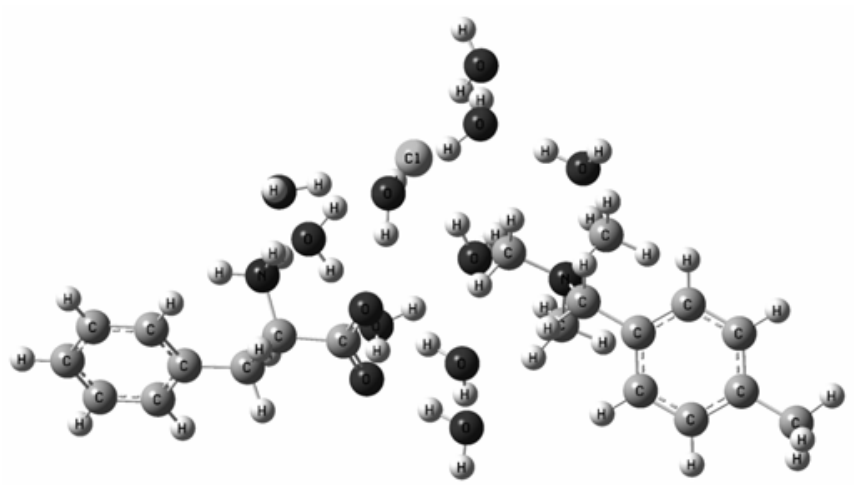

6

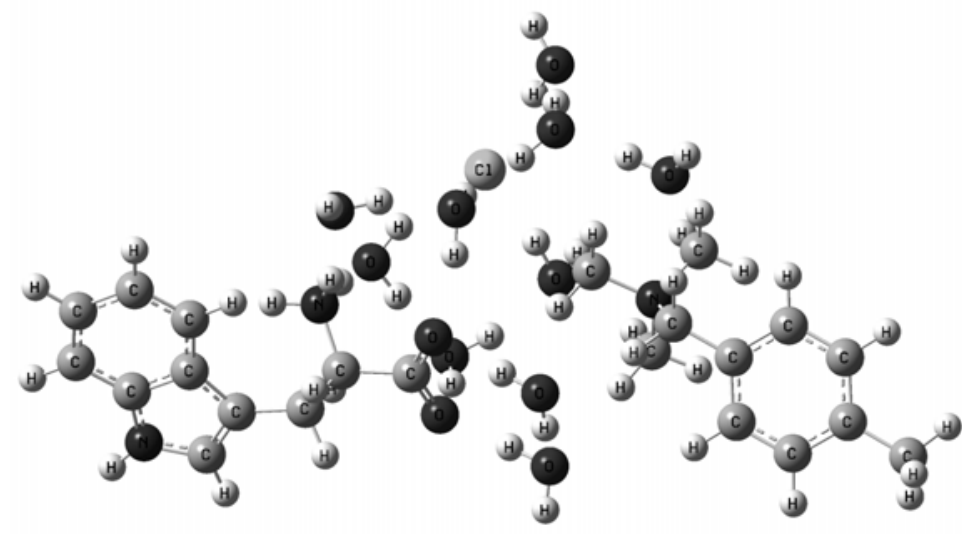

B

Рис. 3. Оптимизированные структуры $\mathrm{AB}-17(\mathrm{HCl})+\mathrm{Gly}^{ \pm}(\mathrm{a}), \mathrm{AB}-17(\mathrm{HCl})+\mathrm{Phe}^{ \pm}$ (б) и $\mathrm{AB}-17(\mathrm{HCl})+\operatorname{Trp}^{ \pm}$(в) с 10 молекулами воды

Рассмотрен ряд структурных, энергетических и спектроскопических характеристик исследуемых систем. В оптимизированных структурах имеются четыре центра локализации избыточного заряда: положительного - атом азота функциональной группы ионообменника и аминогруппы аминокислоты, и отрицательного - противоион хлора и карбоксильная группа аминокислоты. Расположение центров локализации заряда в виде четырехугольника с чередованием положительных и отрицательных зарядов в вершинах, разделенных молекулами воды, свидетельствует о достаточно прочном закреплении аминокислоты в системе за счет кулоновского взаимодействия. Поэтому представляет интерес изменение в зависимости от природы аминокислоты расстояний между противоионом хлора и атомом азота функциональной 
группы анионообменника $\mathrm{r}_{\mathrm{Cl}-\mathrm{Naн}}$, между атомом хлора и атомом азота аминогруппы цвиттерлита $\mathrm{r}_{\mathrm{Cl}-\mathrm{Nak}}$, между атомом азота функциональной группы сорбента и атомом углерода карбоксильной группы аминокислоты $\mathrm{r}_{\mathrm{Naн-Сcooak}}$ Усиление или ослабление силы взаимодействий можно характеризовать по смещению соответствующей полосы колебания в рассчитанном ИК спектре системы [22, 23]. Кроме того, можно оценить выигрыш в энергии при образовании ионно-молекулярных структур в сорбенте $(\Delta \mathrm{E})$ как разность между суммой энергий структурных элементов $\left(\mathrm{E}_{(\mathrm{aн}+5 \mathrm{H} 2 \mathrm{O})+}\right.$ $\left.\mathrm{E}_{(\mathrm{ak}+5 \mathrm{H} 2 \mathrm{O})}\right)$, и энергией оптимизированной структуры $\left(\mathrm{E}_{(\mathrm{aн}+\mathrm{ak}+10 \mathrm{H} 2 \mathrm{O})}\right)$. Данные приведены в таблице 1.

Таблица 1. Некоторые характеристики оптимизированных структур, образующиеся в фазе сорбента при необменной сорбции аминокислот

\begin{tabular}{|c|c|c|c|c|c|}
\hline \multicolumn{2}{|c|}{ Характеристика } & AB-17 (Cl) & $\begin{array}{c}\text { AB-17 (Cl) } \\
+ \text { Gly }+10\end{array}$ & $\begin{array}{c}\text { AB-17 (Cl) } \\
+ \text { Phe }+10\end{array}$ & $\begin{array}{c}\text { AB-17 (Cl) } \\
+\operatorname{Trp}+10\end{array}$ \\
\hline \multirow{3}{*}{$\begin{array}{c}\text { Расстояния } \\
\text { между атома- } \\
\text { ми }\end{array}$} & $\mathrm{r}_{\mathrm{ClN} \text { ak }}, \AA$ & - & 4.88 & 4.90 & 4,91 \\
\hline & $\mathrm{r}_{\mathrm{Cl}-\mathrm{N} \text { ан }}, \AA$ & 4.42 & 5.12 & 5.07 & 4,98 \\
\hline & $\mathrm{r}_{\mathrm{N} \mathrm{aH}^{-}{ }^{-} \text {cooak }}, \AA$ & - & 5.42 & 5.51 & 5,50 \\
\hline \multirow{7}{*}{$\begin{array}{c}\text { Частоты неко- } \\
\text { торых колеба- } \\
\text { ний групп } \\
\text { атомов в рас- } \\
\text { считанном ИК } \\
\text { спектре, } \\
\text { см}^{-1}\end{array}$} & $\mathrm{H}_{2} \mathrm{O}-\mathrm{H}_{2} \mathrm{O}$ & 3416 & 3409 & 3412 & 3411 \\
\hline & 24 & 3473 & 3469 & 3468 & 3469 \\
\hline & Вода у атома хлора & 1725 & 1721 & 1720 & 1719 \\
\hline & $\begin{array}{c}-\mathrm{CH}_{3} \text { функц. групп } \\
\text { анионита }\end{array}$ & $\begin{array}{l}3202 \\
3093\end{array}$ & $\begin{array}{l}3205 \\
3103\end{array}$ & $\begin{array}{l}3202 \\
3077\end{array}$ & $\begin{array}{l}3203 \\
3077\end{array}$ \\
\hline & - $\mathrm{NH}_{3}$ гидратир в ak & - & 2525 & 2596 & 2644 \\
\hline & Вода y - $\mathrm{COO}^{-}$в ak & - & 1714 & 1713 & 1714 \\
\hline & Скелет радикала ak & - & - & $\begin{array}{l}1651 \\
1629\end{array}$ & $\begin{array}{l}1665 \\
1619\end{array}$ \\
\hline \multicolumn{2}{|c|}{$\Delta \mathrm{E}$, кДж/моль } & - & 19.95 & 18.59 & 17.93 \\
\hline
\end{tabular}

Как видно из таблицы, при усложнении строения аминокислоты расстояние между противоионом хлора и атомом азота аминогруппы аминокислоты увеличивается, что свидетельствует об ослаблении связи между ними и подтверждается смещением полосы колебания гидратированной аминогруппы в аминокислоте в сторону больших частот. Это вероятно связано с тем, что наличие ароматического радикала аминокислоты приводит к увеличению электронной плотности на атоме азота аминогруппы, в результате на нем снижается заряд, и электростатическое взаимодействие хлора и азота аминогруппы аминокислоты ослабляется. При этом с усложнением аминокислоты становится меньше расстояние между противоионом хлора и атомом азота функциональной группы сорбента, превышающее, однако, это расстояние в сорбенте при отсутствии цвиттерлита. Необходимо отметить, что расстояние между атомом азота функциональной группы сорбента и атомом углерода карбоксильной группы аминокислоты, а так же частота колебаний гидратированных карбоксильных групп аминокислоты в сорбенте остается неизменной независимо от строения цвиттерлита, что свидетельствует о стабильности этого структурного элемента и его взаимодействий в системе. Также наблюдается неизменность частот колебаний структурных элементов анионообменника и воды в исследуемых системах и появление полос колебания групп атомов аминокислот по сравнению с чистым сорбентом.

Как видно из таблицы, выигрыш в энергии при образовании ионномолекулярных структур в сорбенте $(\Delta \mathrm{E})$ уменьшается при увеличении радикала и его гидрофобности в ряду глицин - фенилаланин - триптофан, однако изменение по сравнению с глицином не превышает 10\%. Это свидетельствует о доминировании

Tрунаева и др. / Сорбционные и хроматографические процессы. 2018. Т. 18. № 2 
полярных взаимодействий в системе по амино-карбоксильным группировкам, которые ослабляются из-за перераспределения электронной плотности и падения заряда на аминогруппе цвиттерлита при усложнении ароматического радикала, и вовлечением аминокислоты в дополнительные гидрофобные взаимодействия.

\section{Заключение}

Таким образом, основными действующими силами в фазе сорбента при необменной сорбции аминокислот являютсяион-ионные и ион-дипольные взаимодействия функциональной группы сорбента, противоиона хлора, биполярного иона цвиттерлита друг с другом и молекулами воды. При усложнении строения бокового радикала аминокислоты в исследуемых сорбционных системах с минеральным противоионом $\mathrm{AB}-17\left(\mathrm{Cl}^{-}\right)+\mathrm{AK}^{ \pm}$структурные характеристики и выигрыш энергии меняются незначительно.

\section{Список литературы}

1. Zuyi Tao. // Ion exchange and solvent extraction. 1995. Vol. 12. pp. 353-380.

2. Селеменев В.Ф., Хохлов В.Ю., Бобрешова О.В.Физико-химические основы сорбционных и мембранных методов выделения и разделения аминокислот. Воронеж. Изд-во ВГУ. 2001, 300 с.

3. Хохлова О.Н., Пискунова Е.С., Трунаева Е.С. // «Физико-химические основы ионообменных и хроматографических проиессов (ИОНИТЫ-2011)», сборник трудов ХІІІ Международной конференции. Воронеж. 2011. С. 162-164.

4. Хохлова О.Н., Распопина Н.Г. // Сорбиионные и хроматографические проиессы. 2001. T. 1. № 6. C. 957-967.

5. Хохлова О.Н. // Вестник ВГУ. Серия: Химия. Биология. Фармация. 2015. № 2. С. 42-45.

6. FrischM.J.,TrucksG.W., SchlegelH.B., ScuseriaG.E., RobM.A., CheesemanJ.R., MontgomeryJr. J.A., VtevenT., KudinK.N., BurantJ.C., MillamJ.M., IyengarS.S., TomasiJ., BaroneV., MennucciB., CossiM., ScalmaniG., RegaN., PeterssonG.A., NakatsujiH., HadaM., EharaM., ToyotaK., FukudaR., HasegawaJ., IshidaM., NakajimaT., HondaY., KitaoO., NakaiH., KleneM., LiX., KnoxJ.E., HratchianH.P., CrossL.B., BakkenV., AdamoC., JaramilloJ., GompertsR., StratmannR.E., YazyevO., AustinA.J., CammiR., PomelliC., OchterskiJ.W., AyalaP.Y., $\quad$ MorokumaK., $\quad$ VothG.A., SalvadorP., DannenbergJ.J., ZakrzewskiV.G., DapprichS., DanielsA.D., StrainM.C., FarkasO., MalickD.K., RabuckA.D., RaghavachariK., ForesmanJ.B., OrtizJ.V., CuiQ., BaboulA.G., Clif-
fordS., CioslowskiJ., StefanovB.B., LiuG., LiashenkoA., PiskorzP., KomaromiI., MartinR.L., FoxD.J., KeithT., Al-LahamM.A., PengC.Y., NanayakkaraA., ChallacombeM., GillP.M.W., JohnsonB., ChenW, WongM.W., GonzalezC., PopleJ.A. Gaussian 03, revisionC.02. PittsburghPA: Gaussian, Inc., Wallingford, CT, 2003.

7. Иониты. Каталог. Черкассы. НИИТЭХим. $1980.32 \mathrm{c.}$

8. Гребенюк В.Д., Мазо А.А. Обессоливание воды ионитами. М. Химия. 1980. 256 c.

9. Иониты в химической технологии. Ред. Б.Н. Никольский, П.Г. Романков. Л. Химия. $1982.416 \mathrm{c.}$

10.Зеленковский В.М., Безъязычная Т.В., Солдатов В.С. // Доклады Национальной академии наук Беларуси. 2003. Т. 47. № 6. С. 44.

11.Мироненко Н.В., Брежнева Т.А., Бутырская Е.В., Селеменев В.Ф. // Журнал общей химии. 2012. Т. 82. Вып. 9. С. 1505-1510.

12.Козадерова О.А. // Мембраныл. 2008. Т. 37. № 1. С.3.

13.Ланцузская Е.В., Орос Г.Ю., Крисилов А.В., Селеменев В.Ф.// Сорбиионные и хроматографические проиессы. 2014. Т. 14. № 4. C. 635-641.

14.Бутырская Е.В., Нечаева Л.С., Шапошник В.А., Дроздова Е.И. // Сорбиионные и хроматографические прочессы. 2012. Т. 12. № 4. C. 501-512.

15.Vysotsky Yu.B., Fomina E.S., Belyaeva E.A., Aksenenko E.V. // J. Phys. Chem. 2011. Vol. 115 (10). pp. 2264-2281. 
16.Kereselidze J., Kvaraia V., Mikuchadze G. // Computational Molecular Bioscience. 2014. No 4. pp. 35.

17.Трунаева Е.С., Хохлова О.Н., Хохлов В.Ю. // Журнал структурной химии. 2015. Т. 56. № 6. C. 1111-1115.

18. RajabiKhadijeh, Fridgen Travis D. // $J$. Phys. Chem. 2008. Vol. 112 (1). pp. 23-30.

19.Тиноко И., Зауэр К., Вэнг Дж., Паглиси Дж. Физическая химия. Принципы и применение в биологических науках. М. Техноcфера. 2005.744 c.

\section{References}

1. Zuyi Tao., Ion exchange and solvent extraction, 1995, Vol. 12, pp. 353-380.

2. Selemenev V.F., Khokhlov V.Yu., Bobreshova O.V., Fiziko-khimicheskie osnovy sorbtsionnykh i membrannykh metodov vydeleniya i razdeleniya aminokislot, Voronezh, Izdvo VGU, 2001, $300 \mathrm{p}$.

3. Khokhlova O.N., Piskunova E.S., Trunaeva E.S., «Fiziko-khimicheskie osnovy ionoobmennykh i khromatograficheskikh protsessov (IONITY-2011)», sbornik trudov XIII Mezhdunarodnoi konferentsii, Voronezh, 2011, pp. 162-164.

4. Khokhlova O.N., Raspopina N.G., Sorbtsionnye i khromatograficheskie protsessy, 2001, Vol. 1, No 6, pp. 957-967.

5. Khokhlova O.N., Vestnik $V G U$. Seriya: Khimiya. Biologiya. Farmatsiya, 2015, No 2, pp. 42-45.

6. FrischM.J.,TrucksG.W., SchlegelH.B., ScuseriaG.E., RobM.A., CheesemanJ.R., MontgomeryJr. J.A., VtevenT., KudinK.N., BurantJ.C., MillamJ.M., IyengarS.S., TomasiJ., BaroneV., MennucciB., CossiM., ScalmaniG., RegaN., PeterssonG.A., NakatsujiH., HadaM., EharaM., ToyotaK., FukudaR., HasegawaJ., IshidaM., NakajimaT., HondaY., KitaoO., NakaiH., KleneM., LiX., KnoxJ.E., HratchianH.P., CrossL.B., BakkenV., AdamoC., JaramilloJ., GompertsR., StratmannR.E., YazyevO., AustinA.J., CammiR., PomelliC., OchterskiJ.W., AyalaP.Y., MorokumaK., VothG.A., SalvadorP., DannenbergJ.J., ZakrzewskiV.G., DapprichS., DanielsA.D., StrainM.C., FarkasO., MalickD.K., RabuckA.D., RaghavachariK., ForesmanJ.B., OrtizJ.V., CuiQ., BaboulA.G., CliffordS., CioslowskiJ., StefanovB.B., LiuG., LiashenkoA., PiskorzP., KomaromiI., MartinR.L., FoxD.J., KeithT., Al-LahamM.A., PengC.Y., NanayakkaraA., ChallacombeM., GillP.M.W.,
20.Селеменев В.Ф., Чиканов В.Н., Фрелих П. // Журнал физической химии. 1990. Т. 64. № 12. C. 3330-3337.

21.Углянская В.А., Чикин Г.А., Селеменев В.Ф. Инфракрасная спектроскопия ионообменных материалов. Воронеж. Изд-во ВГУ. 1989. 208 с.

22.Нечаева Л.С., Бутырская Е.В., Шапошник В.А, Селеменев В.Ф. Структурный анализ катионообменников. Воронеж. Издательско-полиграфический центр «Научная книга». 2012. 163 с.

JohnsonB., ChenW, WongM.W., GonzalezC., PopleJ.A. Gaussian 03, revisionC.02. PittsburghPA: Gaussian, Inc., Wallingford, CT, 2003.

7. Ionity. Katalog, Cherkassy, NIITEKhim, 1980, $32 \mathrm{p}$.

8. Grebenyuk V.D., Mazo A.A. Obessolivanie vody ionitami, M., Khimiya, 1980, 256 p.

9. Ionity $\mathrm{v}$ khimicheskoi tekhnologii, Red. B.N. Nikol'skii, P.G. Romankov, L., Khimiya, 1982, $416 \mathrm{p}$.

10. Zelenkovskii V.M., Bez"yazychnaya T.V., Soldatov V.S., Doklady Natsional'noi akademii nauk Belarusi, 2003, Vol. 47, No 6, pp. 44.

11. Mironenko N.V., Brezhneva T.A., Butyrskaya E.V., Selemenev V.F., Zhurnal obshchei khimii, 2012, Vol. 82, No 9, pp. 1505-1510.

12. Kozaderova O.A., Membrany, 2008, Vol. 37, No 1, pp. 3.

13. Lantsuzskaya E.V., Oros G.Yu., Krisilov A.V., Selemenev V.F., Sorbtsionnye i khromatograficheskie protsessy, 2014, Vol. 14, No 4, pp. 635-641.

14. Butyrskaya E.V., Nechaeva L.S., Shaposhnik V.A., Drozdova E.I., Sorbtsionnye $i$ khromatograficheskie protsessy, 2012, Vol. 12, No 4, pp. 501-512.

15. Vysotsky Yu.B., Fomina E.S., Belyaeva E.A., Aksenenko E.V., J. Phys. Chem., 2011, Vol. 115 (10), pp. 2264-2281.

16. Kereselidze J., Kvaraia V., Mikuchadze G., Computational Molecular Bioscience, 2014, No 4, pp. 35.

17. Trunaeva E.S., Khokhlova O.N., Khokhlov V.Yu., Zhurnal strukturnoi khimii, 2015, Vol. 56, No 6, pp. 1111-1115.

18. Rajabi Khadijeh, Fridgen Travis D., $J$. Phys. Chem, 2008, Vol. 112 (1), pp. 23-30. 
19. Tinoko I., Zauer K., Veng Dzh., Paglisi Dzh., Fizicheskaya khimiya. Printsipy i primenenie v biologicheskikh naukakh, M., Tekhnosfera, 2005, $744 \mathrm{p}$.

20. Selemenev V.F., Chikanov V.N., Frelikh P., Zhurnal fizicheskoi khimii, 1990, Vol. 64, No 12, pp. 3330-3337.

21. Uglyanskaya V.A., Chikin G.A., Selemenev V.F., Infrakrasnaya spektroskopiya io-

Трунаева Евгения Сергеевна - аспирант кафедры аналитической химии, Воронежский Государственный Университет, Воронеж.

Хохлова Оксана Николаевна - доцент кафедры аналитической химии, к.Х.н., Воронежский Государственный Университет, Воронеж.

Хохлов Владимир Юрьевич - профессор кафедры аналитической химии, д.Х.н., Воронежский Государственный Университет, Воронеж. noobmennykh materialov, Voronezh, Izd-vo VGU, 1989, $208 \mathrm{p}$.

22. Nechaeva L.S., Butyrskaya E.V., Shaposhnik V.A, Selemenev V.F., Strukturnyianalizkationoobmennikov, Voronezh, Izdatel'skopoligraficheskiitsentr «Nauchnayakniga». 2012, $163 \mathrm{p}$.

Trunaeva Eugenia S. - postgraduate student, department of analytical chemistry, Voronezh State University, Voronezh. e-mail: evgeniya.tru@bk.ru

Khokhlova Oksana N. - associate professor, department of analytical chemistry, Voronezh State University, Voronezh, e-mail:okxox@yandex.ru

Khokhlov Vladimir Yu. - professor, department of analytical chemistry, Voronezh State University, Voronezh,e-mail: vladkh70@mail.ru 